\title{
Posture Switching for Prolonging Functional Electrical Stimulation Standing in Paraplegic Patients ${ }^{\star}$
}

\author{
A. Krajl, D.Sc., ${ }^{1 / 3}$ T. Bajd, D.Sc., ${ }^{1}$ R. Turk, M.D. ${ }^{2}$ and H. Benko, PPT. ${ }^{2}$ \\ ${ }^{1}$ Faculty of Electrical Engineering and ${ }^{2}$ Rehabilitation Institute, Edvard Kardelj \\ University, Ljubljana, Yugoslavia. ${ }^{3}$ Visiting Professor, Pritzker Institute of \\ Medical Engineering, Illinois Institute of Technology, Chicago, Illinois 60616, \\ U.S.A.
}

\section{Summary}

The presently employed posture for Functional Electrical Stimulation (FES) assisted standing in spinal cord injury (SCI) patients utilises the active locking of knee joints by tetanically stimulating the quadriceps muscle. The hip joints are in hyperextension and the ankle joints remain free. The upper limbs are used for balancing. This posture requires minimal corrective forces exerted by the hands, the weight is transferred across the legs, while very limited forward-backward sway is permitted. Knee jack-knifing may occur in the instance when the gravity knee bending moment exceeds the moment generated by the quadriceps muscle. Because of these effects and fatiguing of the M. quadriceps the standing time is short, lasting from several minutes up to an hour, depending on the condition of the patient's muscles. Cyclical FES with a duty cycle of 10-20 seconds (on/off) fatigues muscle considerably less. The use of different postures for standing requiring activation of different muscles permits the application of cyclical FES. The cyclic FES results in prolonging standing times by a factor of two to five. Utilising this improvement some patients can stand for up to five hours at a time. This functional achievement is comparable to the standing time of a normal man. The incorporation of the principle of posture switching also prolongs standing in patients with weak muscles, because of the possibility of cyclical coactivation of different synergistic muscle assemblies. It is shown that posture switching can be carried out automatically and that patients adapt quickly to it. In addition using co-contraction of two or three muscles with posture switching does further expand the range of suitable patients and improves standing with an increased permissible range of body sway. Also, the FES antigravity action obtained raises hopes for substantially improving FES induced reciprocal gait.

Key words: Paraplegia; Functional electrical stimulation; Posture switching; Prolonged standing.

\section{Introduction}

The rehabilitation of functional mobility of the spinal cord injured patient is * Read at the Scientific Meeting of the International Medical Society of Paraplegia, September 1985, in Edinburgh, Scotland. 
focussed on the use of the wheelchair. Functional electrical stimulation (FES) may at present be applied as an adjunct for increasing the locomotor ability of SCI patients. FES as part of rehabilitation has a long history, Cybulski (1984). Recent feasibility demonstrations in selected thoracic paraplegic patients using FES to restore reciprocal gait assisted by balancing aids such as crutches, walkers, etc. have proven the usefulness of FES and the wonderful possibility for the natural-like restoration of locomotor abilities, $\operatorname{Kralj}(1979,1980,1983)$, Marsolais (1983, 1985), Petrofsky (1983), Thoma (1983) and Bajd (1983, 1984). Whether using surface, percutaneous, or implanted FES electrodes, only very limited restoration of locomotor functions have been reported. Most authors report achieving useful function in the order of a few minutes to 30-60 minutes, for the restoration of standing, $\mathrm{Kralj}$ (1984), Bajd (1982, 1984), and for gait, similar achievements were obtained. Because walking is itself a most important function, most current FES research efforts concentrate on this problem. Research efforts also need to be focussed on the problems of prolonging and improving standing abilities, because this is an indisputable prerequisite for walking. The incorporation of a closed-loop controller for electrically stimulated standing did not, as one would expect, prolong the standing time ( 5 minutes were achieved), but it did improve the standing quality in regard to disturbance resistance, Marsolais (1984), Chizek (1985). The longest standing time reported (30-60 minutes) was achieved by using surface electrodes to bilaterally activate the M. Quadriceps muscles to lock the knee joints. This work describes the advances in the application of the posture switching principle, Kralj (1982), Nashner (1985) enabling FES prolonged standing in SCI patients together with the possible control modes and the results that are obtained.

\section{The fatiguing of FES Muscles}

Regardless of the type of stimulation mode and electrodes employed, the electrically stimulated muscle will fatigue. The fatiguing may be decreased substantially after the muscles have been restrengthened by FES (Brindley, 1978, Kralj, 1983, 1984). Normally, the fatiguing time represents the time elapse from the onset of FES triggered muscle force until the force declines to a specified level (for instance to $50^{\circ}{ }_{0}$ of the initial force). The time elapse is noted as the $50^{\circ}$ o fatiguing time. The latter may last from about 90 seconds up to a few minutes for paraplegic muscles which have not been restrengthened. After FES restrengthening, the $50^{\circ}$ o fatiguing time increases and durations of one minute to about around 10-20 minutes are obtained depending on the patient's initial muscle condition, stage of muscle atrophy and the neurological damage. In principle lower stimulation frequencies will produce less force but also decreased fatiguing. As a compromise stimulation frequencies around $20 \mathrm{~Hz}$ are used. The use of lower frequencies (like $10 \mathrm{~Hz}$ ) are limited by the fusion frequency, tolerable ripple and request for tetanic contraction. Antigravity functions like standing or holding an object in the hand require given uninterrupted muscle forces for a long time. FES researchers have employed different FES modes to obtain long lasting forces from muscles. Peckham (1977) utilised sequential time divided stimulation via multiple muscle electrodes at subfusion frequencies to obtain long lasting forces at a trade-off of complex hardware and 


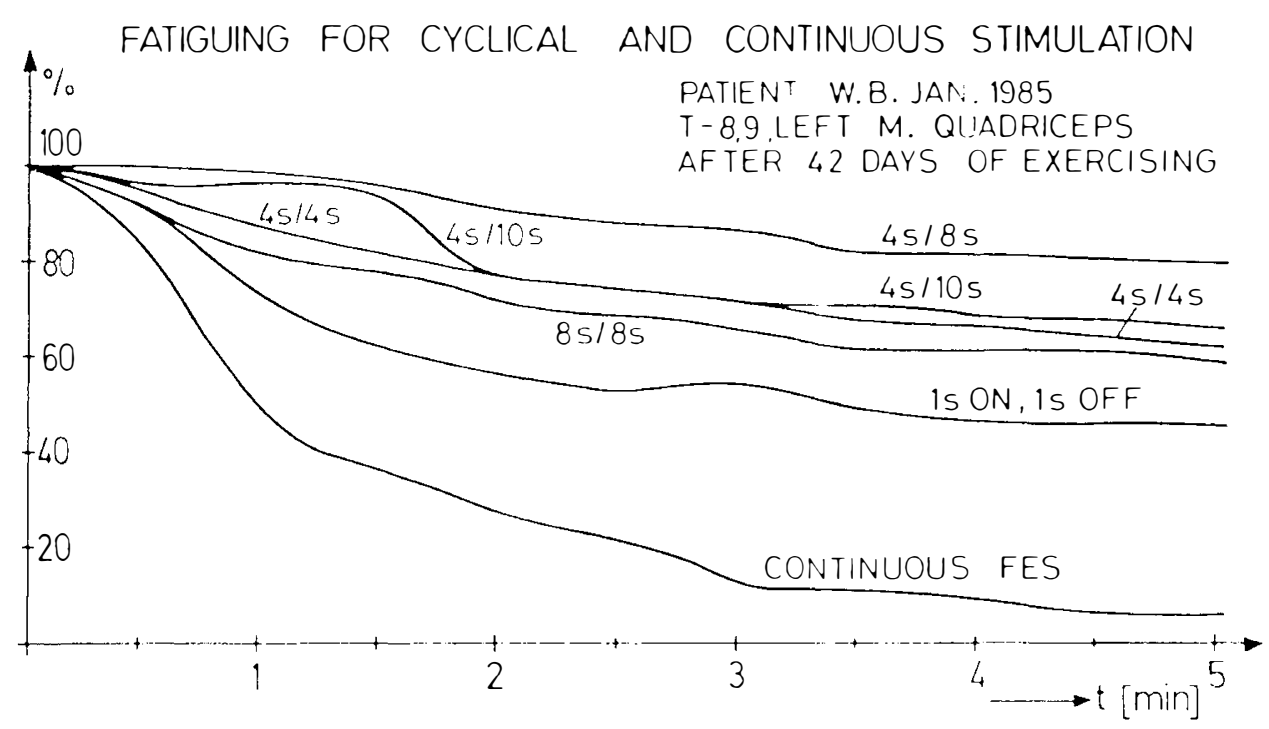

Figure 1. Fatiguing versus time for different stimulation modes. $20 \mathrm{~Hz}$ and $0.3 \mathrm{~ms}$ rectangular stimulation pulses were used. FES was applied using water soaked surface electrodes.

reduced peak force produced. Similarly, Brindley (1978) applied electrodes on three branches of the femoral nerve and cyclically stimulated the corresponding M. Quadriceps heads, thus allowing resting and recovery during the off time. Brindley used $2 \mathrm{~min}$ or $4 \mathrm{~min}$ activation versus 1 or $2 \mathrm{~min}$ resting time. Owing to long recovery times (estimated to be at least 3-5 hours), in Brindley's case the partial fatiguing summed, and the fatiguing was not substantially diminished as one would expect. Our results and published data indicate that cyclical FES activation does in principle decrease fatiguing as shown in Figure 1. Our measurements also confirmed that duty cycles with ON time of 1,2 , or 3 times greater compared to the OFF time will fatigue the muscle in a manner similar to continuous activation. In contrast, the prolongation of OFF time does decrease fatiguing.

Summarising the findings, it is evident that regardless of the FES activation mode, fatigue is a cumulative process which may be slowed by incorporating cyclical stimulation with resting periods and by using the lowest stimulation frequencies possible.

Standing is a function providing permanent anti-gravity support to the body thus requiring constant locking of joints. The latter is provided by muscle action. In the case of insufficient muscle action, the moments due to gravity overcome the locking and collapse of the human standing structure. At this instance the standing is abruptly cut-off. Consequently FES enabled standing is limited because of rapid fatiguing of muscles. To achieve prolonged standing, the idea of posture switching was proposed, Kralj (1982). Normal people when standing for prolonged periods of time frequently make use of changes of posture. Doing so eases the 'effort', by providing resting periods for the muscles thus making use of the less fatiguing cyclical activation. Similarly by using FES one can provide resting periods for muscles and also incorporate the less fatiguing cyclical 
stimulation. We have described the idea or principle of posture switching. As shown in Figure 2A, the common used posture for FES assisted standing employs bilateral FES of $M$. quadriceps for knee locking, hips are hyperextended and locked by ligaments while the ankle joints are stabilised by providing balancing forces exerted by the hands on the crutches or walker. Obviously using the posture displayed in Figure 2B, the patient is able to stand. Here the soleus and gastrocenemius muscles are stimulated. The soleus muscle according to our measurements has nearly double the fatiguing time compared to the quadriceps. consecutive activation between the described postures given in Fig. 2A and 2B will at least double the standing time because two muscle groups are used. An additional prolongation of standing time results if cyclical stimulation is introduced. Extending the posture switching principle by including the hip extensor muscles will additionally increase the standing time. The principle of muscle synergism, if introduced at that moment, permits standing by patients who have weak muscles because the action of two or all three synergistic extensor muscles can be used in combination. Note that the latter is very important particularly in SCI, since multiple trauma is common and the extensor muscles are usually involved and further weakened because of disuse atrophy. Figure 3 displays a possible posture switching sequence and also indicates that the activities of different muscles may be carried out unilaterally, bilaterally, and mixed, resulting in longer off times and thus additionally prolonging the standing time. We note that each posture requires specific mechanical prerequisites with regard to the passing of the centre of gravity line to the joints and also requires adequate mobility $(\mathrm{ROM})$ of the joints. If the latter constraints are not met, posture switching is not possible or even dangerous collapsing may occur.

\section{Practical use by patients}

First the postures shown in Figs. 2 and 3 have been tested. SCI patients suffering complete lesions from T4-T10 were selected. Some of them completed the FES restrengthening and some did not. All the given postures proved to be useful using bilateral FES and patients were able to stand employing both of the postures shown in Fig. 2. Mixed activation of muscles provided satisfactory standing while postures using unilateral FES were not applicable to every patient. The coactivation of synergistic muscles also proved very useful during standing upright. Patients reported that, standing-up was easier requiring less reliance upon the hands. For these experiments the stimulator used by Bajd (1983) was modified by incorporating switch triggering of each of four channels. For M.gluteus maximus stimulation an additional 2-channel PARA 1 stimulator was used. (PARA 1 is a trade name of a commercially available stimulator intended for muscle restrengthening and functional standing. The stimulator is

produced by SOCA-Rehabilitation Institute Ljubljana, Yugoslavia). While switching FES of muscles during standing, the ground reaction vector and its displacement were recorded together with stimulation artefact measured with EMG electrodes which provided a record of FES activity versus time. During the first trials the switching was performed by a third person who notified the patient when switching was actually taking place. Later the switching was performed by the patient himself. After a few days of use random switching was 


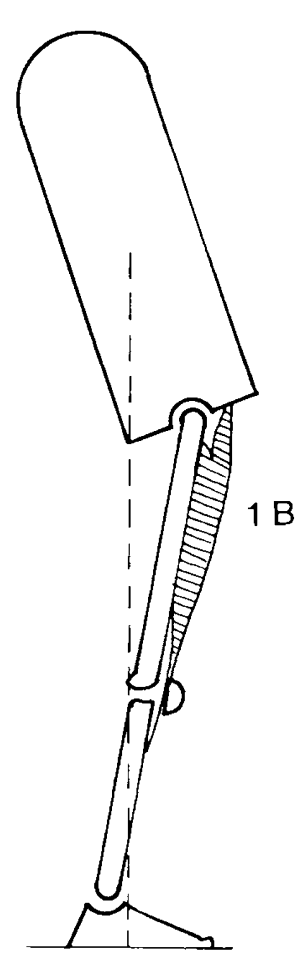

A

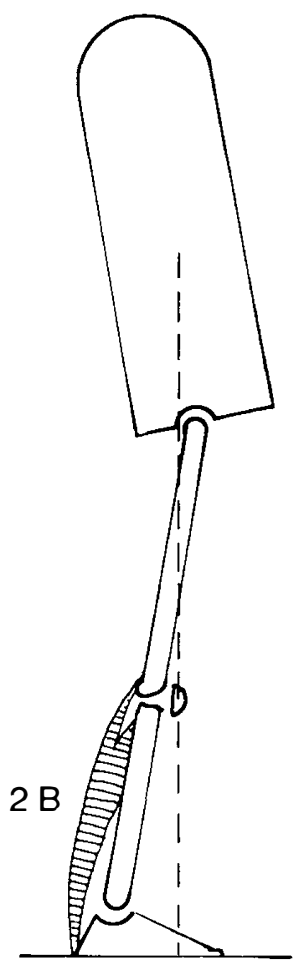

B

Figure 2. The commonly used standing posture requiring bilateral FES of $M$. quadriceps is shown in $\mathrm{A}$, while the posture for $\mathrm{M}$. soleus and $\mathrm{M}$. gastrocnemius standing is given in $\mathrm{B}$. Note the difference in the direction of the centre of gravity line.

introduced. This was to test the hypothesis if automatic switching or arbitrarily selected switching by the physical therapist to change postures may also be feasible. Affirmative results may have far reaching consequences, allowing the use of prolonged standing of patients in selected postures without requiring any control by the patient.

\section{Results}

The M. quadriceps standing posture has been used in Ljubljana in over 50 patients. The M.soleus and M.gastrocnemius posture, Fig. 3B, was in the last two years tested in more than 10 patients. This posture is not applicable for a number of patients because of progressed ankle joint plantar flexion contractures, weak calf muscles, or very spastic plantar flexor muscles. It is interesting to note, that some patients are able to maintain the ' $C$ ' posture easier by using the Fig. 3C posture, because of absent flexion torque at the hip which is normally caused by FES M.rectus femoris. The posture of Fig. 3D was verified and nearly all patients able to assume the Fig. 3C posture. To date we have studied three patients who were unable to stand by FES activation of only one muscle group, because of insufficient locking torque produced by the muscle. By employing the standing posture in Fig. 3D these patients were able to stand for several 


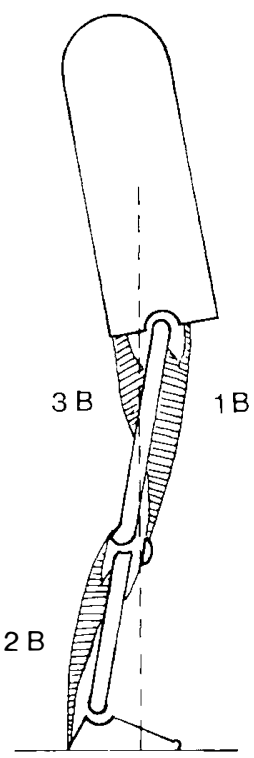

$A$

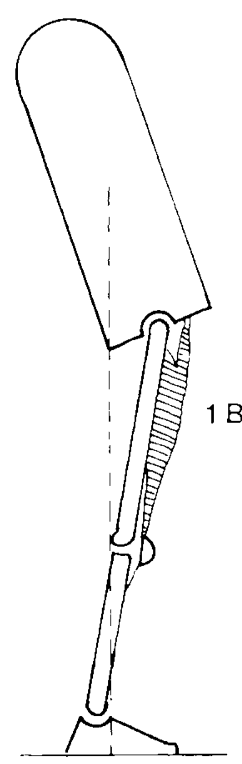

$B$

1.M. QUADRICEPS FEMORIS

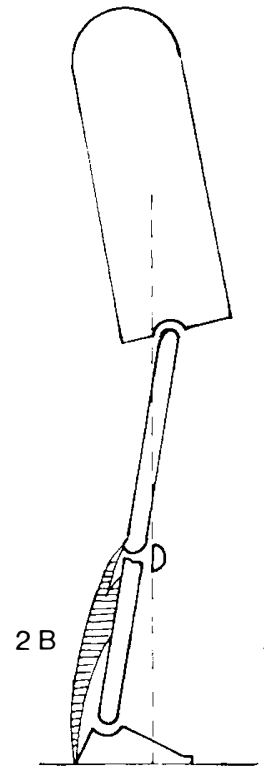

C

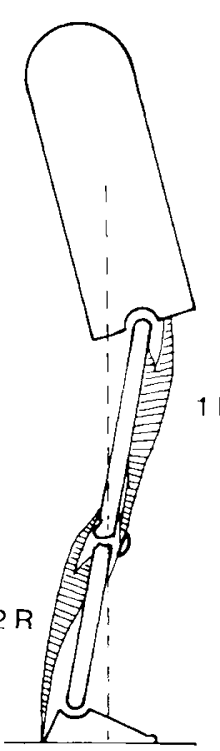

$D$

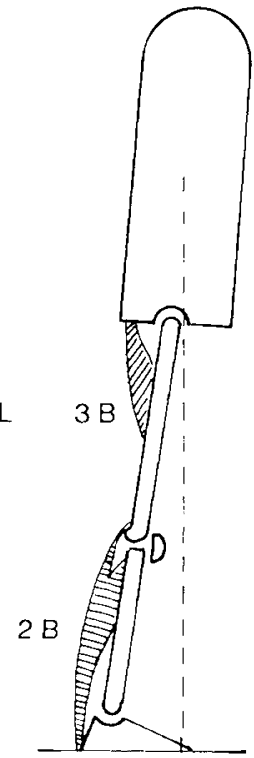

E

\section{M. GASTROCNEMIUS AND SOLEUS \\ $L-L E F T$ \\ 3.M. GLUTEUS MAXIMUS AND MEDIUS R-RIGHT}

$B-B I L A T E R A L$

Figure 3. A possible posture switching sequence is presented. In $B$ and $C$ are given the two basic modes while in $\mathrm{E}$ the posture for synergistic standing by FES of $\mathrm{M}$. gluteus maximus and medius and the calf muscles is presented. Figure 3D displays the synergistic standing as used for weak muscles and as a transition state during switching from $3 \mathrm{~B}$ to $3 \mathrm{C}$ and vice versa. Figure 3A posture employs FES of all main extensors muscles. This mode is useful for standing-up and in patients with weak muscles. Note that some of the given postures may be obtained also by unilateral stimulation and consequently of mixed left/right selection of different muscles.

minutes. It is interesting to note that patients with strong knee and plantar flexor muscles indicated that during the standing-up manouver the 'pushing-up' of their extremities eased the necessary assistance usually provided by hands. We did not analyse this in detail biomechanically.

The posture in Fig. 3E was tried in only a few patients, mainly because of the inconvenient and tedious electrode attachment procedure required. This posture also proved to be useful and patient's remarks were similar to those using the posture shown in Fig. 3D, regarding the redirection of hand involvement. In most of our experiments we used On/Off switching of the required stimulation channels, while for regular use we utilise gradual ramping transitions. The unilateral postures, in which only one site muscles are activated did not prove to work well. Patients required to use their hands to adjust the body weight to the site that the support will be provided. Thus increased hand forces are required. Also they did not know in advance the degree of support and when to be ready to prevent medial dropping. Only one patient who uses the four 
channel stimulator daily for walking was able to manage the postures using unilateral stimulation of muscles to a high degree of satisfaction.

For smoother transfers among postures it is preferable to use ramping of the stimulation train. We selected 1-2 s. ramps, but did not study the most favourable ramping times. To ensure safety, the Off switching ramping started after the complete onset of amplitude in the On coming channel. The best On/Off timing should be elaborated in detail and with regard to the muscles used. The experiments have not been optimised, yet standing times have in general at least doubled because of the employment of posture switching as in the Fig. 3B and 3C posture switching. Therefore standing times of 30 minutes and up to 60 minutes were obtained in patients who were considered to be poor at standing: Bajd (1984). For patients capable of standing for 30 or 60 minutes only by $M$. quadriceps stimulation the former switching mode if used has prolonged the standing times for up to 3 hours, and in two patients we were able to produce a standing time of nearly 5 hours, thus approaching the performance of a normal man while standing. 'Marathon' standing performance testing using the postures in Fig. $3 \mathrm{E}$ and Fig. 3A is in progress. So far the results indicate that posture switching may prove to be an effective and important mode of FES application to ensure prolonged standing in SCI patients. Therefore detailed biomechanical, performance and control studies are indicated. The results of verifying if automatic control may be applicable are affirmative. Fig. 4 presents a record

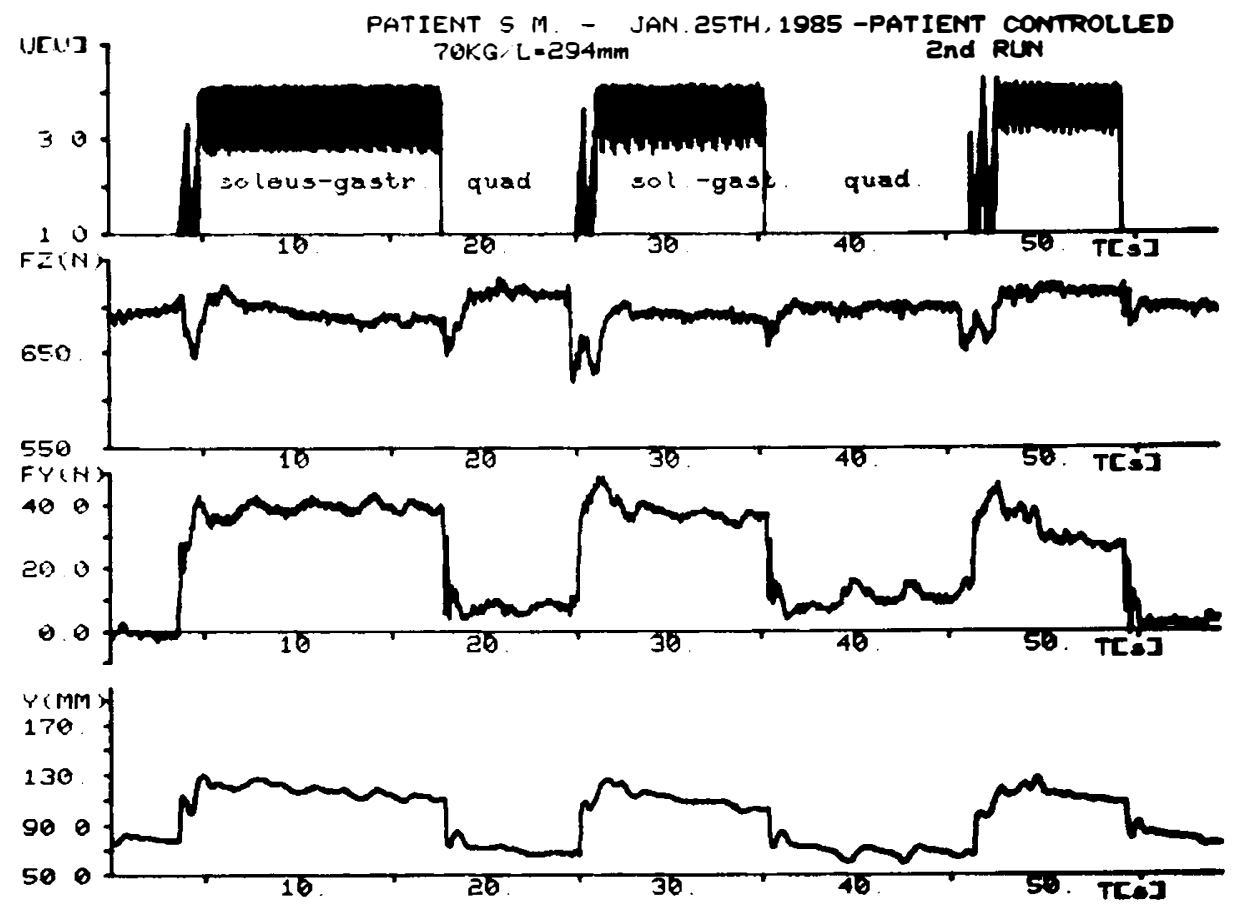

Figure 4. Patient controlled posture switching for Figures $2 \mathrm{~A}$ to $2 \mathrm{~B}$ posture exchange. The upper trace is the record of FES sequencing, the second trace is the FZ $(\mathrm{N})$-vertical ground reaction force in Newtons, measured by the force plate. Third trace FY (N) - is the anteroposterior shear force in Newtons and the fourth trace $\mathrm{Y}(\mathrm{MM})$ shows the displacement of the supporting vector in the $\mathrm{A} / \mathrm{P}$ direction. Higher values of $\mathrm{Y}$ correspond to anterior sway. 


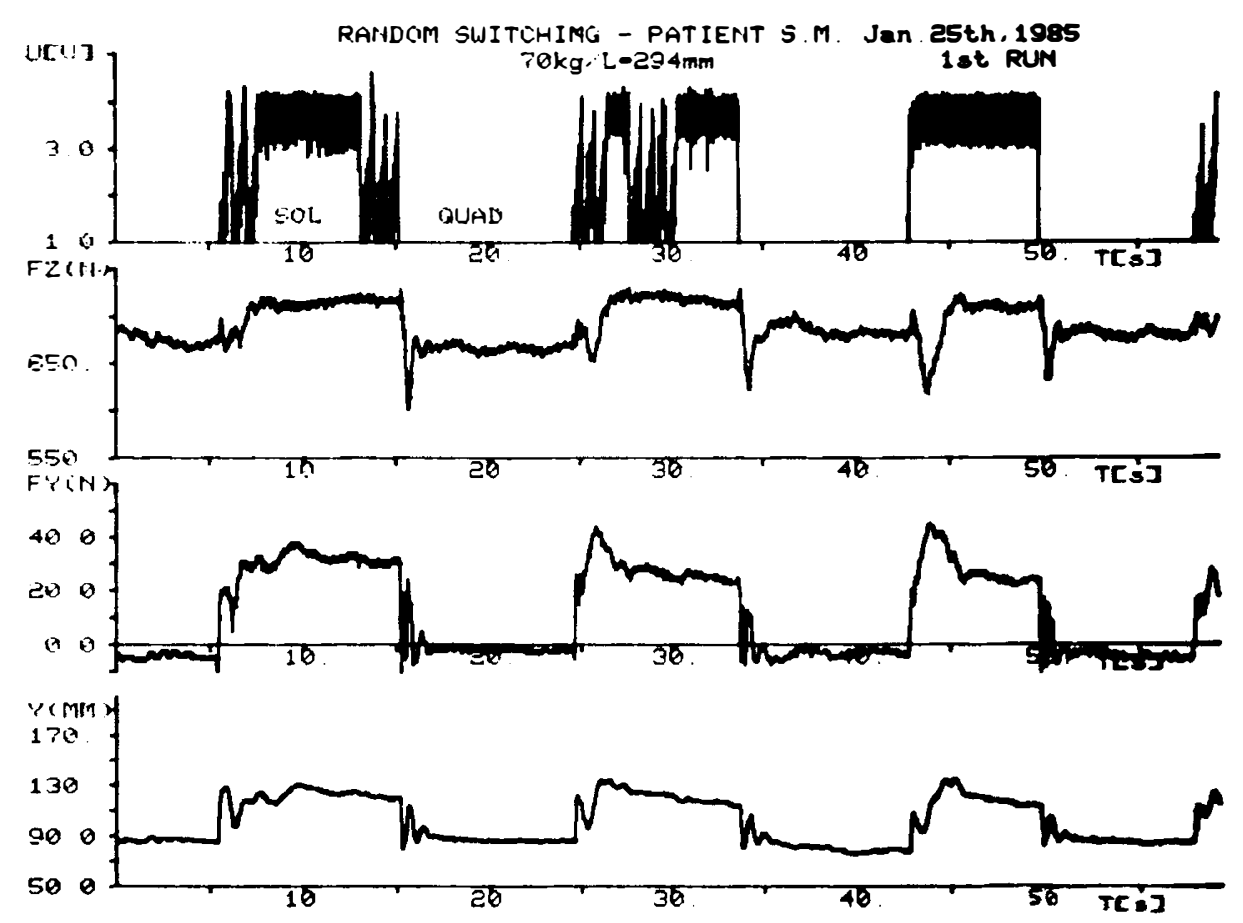

Figure 5. Random switching without patient awareness applied for posture changing according to Figures $2 \mathrm{~A}$ and $\mathrm{B}$. The data have been recorded with the same channel calibration and assignment as in Figure 4, for easier comparison of performance shown in Figure 4 with Figure 5.

for posture switching for the postures given in Figs $3 \mathrm{~B}$ and $3 \mathrm{C}$. The patient controlled the switching by means of switches attached to a roller walker. On/Off switching was used and the upper trace in Fig. 4 displays stimulation timing. The FZ $(\mathrm{N})$ trace is the record of vertical reaction force, $\mathrm{FY}(\mathrm{N})$ the record of $\mathrm{A} / \mathrm{P}$ shear force, the ground reaction vector location and $\mathrm{Y}(\mathrm{mm})$, given in millimetres in the $\mathrm{A} / \mathrm{P}$ direction. Higher values in FY and $\mathrm{Y}$ indicate anterior sway. In Fig. 4 it can be seen that the patient learned after a few switching manouvres to adjust his upper body position according to the leg support provided. In this case a T5, 6 complete injured paraplegic patient was using FES. At the times correlated with the switching instants we see in the FZ trace a decline of force showing that the patient is adjusting himself by his hands his truck position. But it is evident that the hand involvement is less for each consecutive trial. The biomechanical constraint for $M$. quadriceps and $M$. soleus and gastrocnemius standing is clearly displayed in trace FY and $\mathrm{Y}$. The $\mathrm{M}$. soleus and gastrocnemius standing requires an anterior placed ground reaction vector. In the given case the patient moved the ground reaction vector by about $4 \mathrm{~cm}$ using the hands and trunk movement. If random switching was performed, without the patient knowing when it will take place, a similar record to the one in Fig. 4 was obtained as is shown in Fig. 5. Immediately, one observes the decreased $\mathrm{A} / \mathrm{P}$ shear force. Also involvement of the hands at the switching instance is increased. These changes are not significant and in principle the display in Fig. 5 is quite similar to the results 
shown in Fig. 4. Because of this we may conclude that automatic posture switching is feasible. Of course the detailed control strategy with necessary sensing mode, sensors placement and safety etc needs to be elaborated. Here it is sufficient to note that the 'software' for posture switching and the results obtained can be programmed with the stimulator controller. Any time the patient stands-up the posture switching may be on, while if the patient starts to perform functional tasks while standing, the appropriate posture may be automatically selected by the device depending on the sensory information obtained and logic software stored and additional muscles stimulated, whilst the muscles which are not needed may be gradually ramped off. The posture switching is of great importance because it indicates the feasibility of ensuring an upright posture in SCI patients for time periods comparable to a normal man's performance. In addition the principle of posture switching has far reaching implications, because if utilised with synergistic activation (coordination) in FES gait synthesis it may, as we expect, substantially improve the gait. The former is valid if we are hypothesising that gait is composed of a series of postures.

\section{Summary}

Posture switching for further prolonging FES standing in paraplegic patients has been developed. It is shown that the introduction of posture switching prolong standing by means of FES by a factor of at least 2 times or more. In some patients with good musculature and near normal range of joint movement, standing for up to 5 hours was achieved. Thus results comparable to normal human performance were obtained. It is also shown that posture switching with synergistic activation of FES muscles can be used to enable patients to stand up who have weak muscles. The results indicated that random switching of FES channels for change of postures is possible. The latter enables the employment of automatic control in posture switching. In this regard the exchange of postures is according to some criteria software preprogrammed into the stimulator. It was also shown that if gait is considered to be a series of postures the advances in posture switching may also substantially contribute to the improved synthesis of gait by means of FES. Therefore it is believed that the posture switching principle has far reaching consequences for the development of the FES techniques.

\section{References}

Bajd T, KRALj A, Turk R 1982 Standing of a healthy subject and a paraplegic patient. Journal of Biomechanics 15:1-10.

BAjD T, KRALj A, TURK R, et al. 1983 The use of a four-channel electrical stimulator as an ambulatory aid for paraplegic patients. Physical Therapy 63:1116-1120.

Bajd T, KRALJ A, KRAjnik J, et al. 1984 Standing by FES in paraplegic patients. Proc. 8th Int. Symp. on Ext. Contr. of Human Extremities, Dubrovnik, Yugoslavia, pp. 51-61.

Brindley GS, Polkey CE, Rushton DN 1978 Electrical splinting of the knee in paraplegia. Paraplegia 16: 428-435.

Chizeck HJ, Lalonde R, Chang CW, et al. 1985 Performance of a closed-loop controller for electrically stimulated standing in paralyzed patients. Proc. of the RESNA 8th Ann. Conf., Memphis, Tenn., pp. 231-233.

Cybulski GR, Penn R, Jaeger R 1984 Lower extremity functional neuromuscular stimulation in cases of spinal cord injury. Neurosurgery 15:132-145. 
KRALJ A, BAJD T, TURK R, 1980 Electrical stimulation providing functional use of paraplegic patient muscles. Medical Progress Through Technology 7:3-9.

Kralj A, BAJd T, TURK R, et al. 1979 Paraplegic patients standing by functional electrical stimulation. Digest of 12th Int. Conf. on Medic. Physics, Jerusalem, Israel, paper 59.3.

KRALJ A, BAJD T, TURK R, et al. 1983 Gait restoration in paraplegic patients: A feasibility demonstration using multichannel surface electrode FES. Journal of Rehabilitation and Development, Vol. 20, No. 1, pp. 3-20.

KRALJ A, BAJD T, TURK R, et al. 1981 Experiences with FES enabled standing in complete paraplegic patients. Proc. of the 7th Symp. on Ext. Contr. of Human Extremities, Dubrovnik, Yugoslavia, pp. 279-303.

Kralj A, Grobelnik S 1973 Functional electrical stimulation-A new hope for paraplegic patients? Bulletin of Prosthetics Research, fall, 10-20:75-102.

KRALJ A, JAEger RJ 1982 Posture switching enables prolonged standing in paraplegic patients functionally electrically stimulated. Proc. of the RESNA 5th Ann. Conf., Houston, Texas, p. 60 .

MARSOlias EB, Kobetic R 1985 Kinematics of paraplegic gait produced by electrical stimulation. Proc. of the RESNA 8th Ann. Conf., Memphis., Tenn., pp. 108-110.

Marsolais EB, Kobetic R, Chizek H, et al. 1984 Standing of paraplegics using closed-loop controlled stimulation. Proc. of 8 th int. symp. on external control of human extremities, Dubrovnik, Yugoslavia, pp. 63-65.

Marsolais EB, Kobetic R, Cochoff GF, et al. 1983 Reciprocal walking in paraplegic patients using internal functional electrical stimulation. Proc. 6th Annual Conf. on Rehab. Engineer., San Diego, pp. 78-80.

NASHNer LM, MCCollum G 1985 The organization of human posturalmovements: A formal basis and experimental synthesis. The Behavioral and Brain Sciences 81:135-172.

Peckham H, Mortimer T 1977 Restoration of hard function in the quadriceps through electrical stimulation, Functional electr. stimul. Applications in Neural Prosth., Edts.: Hambrecht FT and Reswick JB, Marcel Dekker, New York, p. 83.

Petrofsky JS, Phillips CA 1983 Computer controlled walking in the paralyzed individual. J. Neur. Orthop. Surg. 4:153-164.

Thoma H, Frey M, Holle J, et al. 1983 Paraplegics should learn to walk with their fingers. Proc. of the 5th Ann. Conf. IEEE Engng. in Medicine and Biol., Columbus, Ohio, pp. 579-582. 